Published in "Physical Chemistry Chemical Physics 16(5): 2011-2019, 2013"

which should be cited to refer to this work.

\title{
Vibronic spectra of the $p$-benzoquinone radical anion and cation: a matrix isolation and computational study $\dagger$
}

\begin{abstract}
Krzysztof Piech, ${ }^{a}$ Thomas Bally, ${ }^{\text {a }}$ Takatoshi Ichino $^{\mathrm{b}}$ and John Stanton*b
The electronic and vibrational absorption spectra of the radical anion and cation of $p$-benzoquinone (PBQ) in an Ar matrix between 500 and $40000 \mathrm{~cm}^{-1}$ are presented and discussed in detail. Of particular interest is the radical cation, which shows very unusual spectroscopic features that can be understood in terms of vibronic coupling between the ground and a very low-lying excited state. The infrared spectrum of $\mathrm{PBQ}^{\bullet+}$ exhibits a very conspicuous and complicated pattern of features above $1900 \mathrm{~cm}^{-1}$ that is due to this electronic transition, and offers an unusually vivid demonstration of the effects of vibronic coupling in what would usually be a relatively simple region of the electromagnetic spectrum associated only with vibrational transitions. As expected, the intensities of most of the IR transitions leading to levels that couple the ground to the very low-lying first excited state of $\mathrm{PBQ}{ }^{\bullet+}$ increase by large factors upon ionization, due to "intensity borrowing" from the $D_{0} \rightarrow D_{1}$ electronic transition. A notable exception is the antisymmetric $\mathrm{C}=\mathrm{O}$ stretching vibration, which contributes significantly to the vibronic coupling, but has nevertheless quite small intensity in the cation spectrum. This surprising feature is rationalized on the basis of a simple perturbation analysis.
\end{abstract}

\section{Introduction}

Molecules containing the $p$-benzoquinone (PBQ) functionality serve as electron "gates" in the primary reactions of photosynthesis and in the mitochondrial respiratory system, ${ }^{1}$ and as general oxidants or hydrogen acceptors in different chemical processes. $^{2}$ Therefore the radical anion of PBQ (also called the semiquinone radical anion) has been investigated quite thoroughly by different spectroscopic techniques. Its excited state structure has been probed by different methods, both in the gas ${ }^{3-6}$ and condensed phases. ${ }^{7-10}$ With regard to the vibrational structure of $\mathrm{PBQ}^{\bullet-}$, several groups have reported and assigned (resonance) Raman spectra which revealed some of the totally symmetric fundamentals, ${ }^{11-15}$ but only the strongest band in the IR spectrum, at around $1500 \mathrm{~cm}^{-1}$, has been unambiguously pinpointed to date. ${ }^{14,16} \mathrm{PBQ}^{\bullet-}$ has also been the subject of several early semiempirical $^{17,18}$ and more recent $a b$ initio theoretical studies. ${ }^{9,19-21}$

On the other hand, much less is known about the PBQ radical cation, probably because $\mathrm{PBQ}$ is rather hard to oxidize.

\footnotetext{
${ }^{a}$ Department of Chemistry, University of Fribourg, CH-1700 Fribourg, Switzerland. E-mail:Thomas.Bally@unifr.ch

${ }^{b}$ Department of Chemistry and Biochemistry, The University of Texas at Austin, Austin, TX 78712-0165, USA. E-mail: jfstanton@gmail.com

$\dagger$ Electronic supplementary information (ESI) available: Full citation of ref 43 . Cartesian coordinates and results of TD-DFT calculations on PBQ, PBQ ${ }^{\bullet-}$, and PBQ ${ }^{\bullet+}$. See DOI: $10.1039 / \mathrm{c} 3 \mathrm{cp} 53960 \mathrm{a}$
}

Most of the discussions on $\mathrm{PBQ}^{\bullet+}$ and its electronic structure have revolved around the proper assignment of the photoelectron (PE) spectrum of $\mathrm{PBQ}$, which was rather hotly debated for two decades. ${ }^{22}$ The problem was that $\mathrm{PBQ}^{\bullet+}$ has two sets of close-lying states, one resulting from ionization out of a pair of proximate $\pi$, the other out of a pair of nearby $\sigma$-MOs, any of which could potentially be the ground state.

It has now been established beyond any doubt that the ground state of $\mathrm{PBQ}^{\bullet+}$ arises by ionization from the orbital labeled $n_{-}$in Fig. 1, which has $b_{3 g}$ symmetry if the cartesian axes are chosen as indicated in this figure. ${ }^{19,23}$ However, it has also been shown that, in order to correctly model the first two bands in the PE spectrum of $\mathrm{PBQ}$, and hence the electronic structure of $\mathrm{PBQ}^{\bullet+}$, vibronic coupling between the proximate states must be accounted for, ${ }^{23}$ an issue that will arise again in the present discussion of its electronic absorption spectrum. To the best of our knowledge, $\mathrm{PBQ}^{\bullet+}$ has never been characterized by any technique other than PE spectroscopy.

In this paper we present the electronic and vibrational absorption spectra of the radical anion and cation of PBQ, obtained in Ar matrices. Whereas the radical anion spectra can be readily modelled using standard methods of quantum chemistry, the very unusual infrared spectrum above $1800 \mathrm{~cm}^{-1}$ of $\mathrm{PBQ}^{\bullet+}$ cannot be explained without accounting for vibronic coupling, which requires a sophisticated treatment of the nonadiabatic system. 

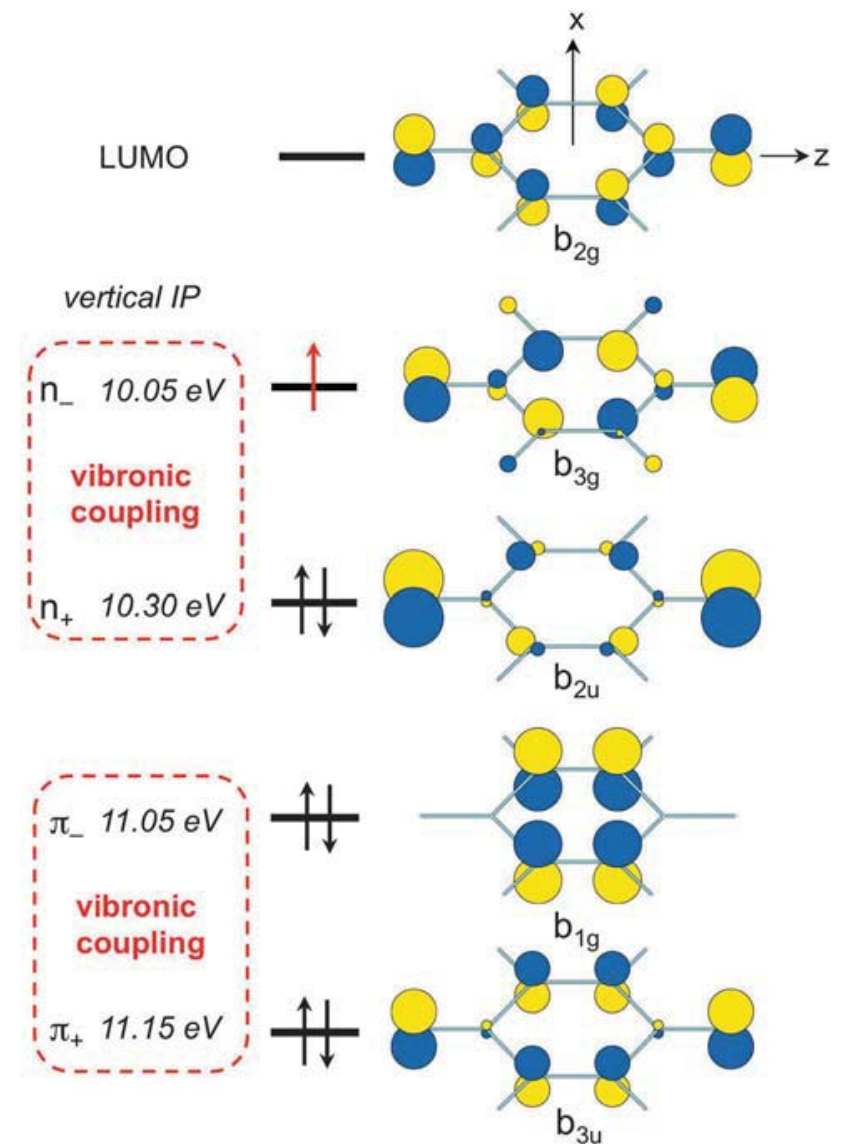

Fig. 1 The highest four occupied and the lowest unoccupied molecular orbitals of PBQ (ionization from each of these orbitals gives rise to a state of the radical cation which has the same symmetry as that of the resulting singly occupied MO). Italicized numbers are vertical energy differences between neutral PBQ and the four ionic states. ${ }^{23}$ Note that pairs of $\sigma$ - and $\pi$-states lie very close in energy, which acts to amplify vibronic coupling effects.

\section{Experimental results}

To obtain radical ions in Ar matrices containing the neutral precursor, these matrices are exposed to X-irradiation. This leads to formation of $\mathrm{Ar}^{\bullet+} / \mathrm{e}^{-}$pairs which ultimately transfer their charge to substrate molecules contained in the matrix. Thus one can generate radical cations, the yield of which can be enhanced by adding an electron scavenger, such as methylene chloride. ${ }^{24}$ This turns out to be unnecessary in the present case because PBQ itself is an excellent electron scavenger. If, however, a more easily oxidizable "hole scavenger" such as 1,4-diazabicyclooctane (DABCO) is added, radical anions of added substrates can be formed, provided that the anions of these substrates are bound in Ar. $^{10,25}$ Here we have exploited this technique to differentiate the spectra of radical cations and anions of PBQ.

The optical spectrum obtained upon X-irradiation of $10 \mathrm{~K} \mathrm{Ar}$ matrices containing $c a$. one part per thousand of PBQ and of DABCO each is dominated by the well-known features of the PBQ radical anion which peak at 458 and $325 \mathrm{~nm}^{7}$ Upon irradiation at $313 \mathrm{~nm}$, the peaks of $\mathrm{PBQ}^{\bullet-}$ disappear,

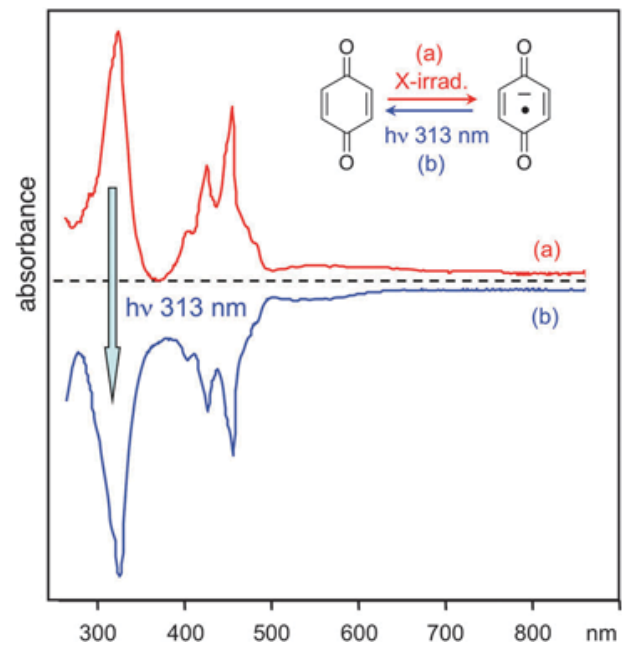

Fig. 2 Electronic absorption spectrum of $\mathrm{PBQ}$ after $\mathrm{X}$-irradiation in $\mathrm{Ar}$ in the presence of $D A B C O$ as a hole scavenger (a); difference spectrum for the bleaching of $\mathrm{PBQ}^{\bullet^{-}}$at $313 \mathrm{~nm}$ (b).

probably due to ejection of the electron which migrates through the matrix and recombines with the positive charge carriers $\left(\right.$ mostly $\left.\mathrm{DABCO}^{\bullet+}\right)$. These processes are documented in Fig. 2. The spectra are very similar, but weaker when no scavenger is added, which indicates that the radical cation of PBQ does not show any substantial absorptions in the energy range that is accessible in this experiment.

In contrast, the mid-IR spectra differ substantially depending on whether or not the matrix is doped with $\mathrm{DABCO}$ in addition to PBQ (compare traces b and c of Fig. 3). The spectrum of PBQ in an Ar matrix (Fig. 3a) is in very good accord with the gas phase and solution spectra ${ }^{26}$ (an assignment will be given in the computational section).

As will be shown below, all of the peaks in the difference spectrum which was obtained on X-irradiation of an Ar matrix containing DABCO in addition to $\mathrm{PBQ}$, can be readily assigned to $\mathrm{PBQ}^{\bullet-}$, based on a simple DFT calculation, with the exception of the intense one at $714 \mathrm{~cm}^{-1}$, which is due to the radical cation of DABCO $\left(\mathrm{D}^{\bullet+}\right)$, two small ones at 801 and $1209 \mathrm{~cm}^{-1}$ (marked with asterisks in Fig. 4b) which will be assigned below to the radical cation of PBQ, and the broad one at $1605 \mathrm{~cm}^{-1}$, which is due to water. On X-irradiation the peaks of neutral PBQ (marked with dashed arrows in Fig. 3) and those of DABCO at 986, 1065, 1324, and $1456 \mathrm{~cm}^{-1}$ (marked "D" in Fig. 3) diminish. On $313 \mathrm{~nm}$ photolysis the processes observed on X-irradiation are mostly reversed (lower spectrum b), except for the peaks of neutral DABCO which are too weak to be seen reappearing.

On the other hand, when no DABCO is added to the matrix, a very different spectrum is obtained on X-irradiation (top spectrum c). As shown below, the three intense peaks at 613, 801 , and $1209 \mathrm{~cm}^{-1}$ and perhaps a small peak at $c a .1655 \mathrm{~cm}^{-1}$ (quite close to a very strong absorption of neutral PBQ) can be assigned to $\mathrm{PBQ}^{\bullet+}$. The presence of the strongest peak of $\mathrm{PBQ}^{\bullet-}$ at $1509 \mathrm{~cm}^{-1}$ indicates that PBQ acts effectively as a scavenger of the electrons that are liberated in the course of X-irradiation (upon careful inspection, some of the weaker bands of $\mathrm{PBQ}^{\bullet-}$ can also be 


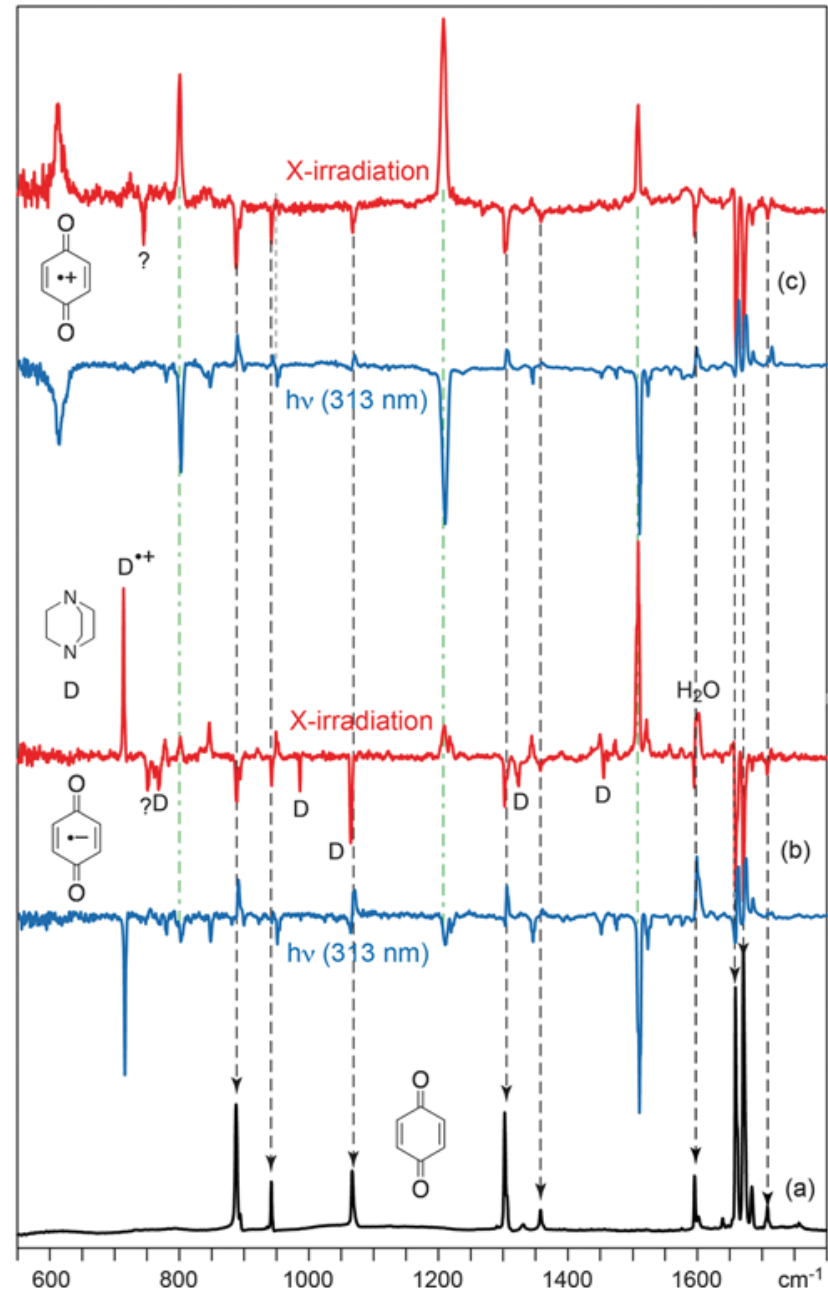

Fig. 3 (a) Spectrum of PBQ in an Ar matrix at $10 \mathrm{~K}$ (b) and (c) IR difference spectra of the species formed on X-irradiation of PBQ in Ar (top traces) and for the subsequent bleaching at $313 \mathrm{~nm}$ (bottom traces); (b) $P B Q$ mixed with an equimolar amount of DABCO (D); (c) PBQ alone. Dashed lines with arrows at the end denote peaks of neutral PBQ, dashed-dotted lines bands which are common to both experiments.

discerned in this spectrum). In view of the high electron affinity of PBQ (1.86 eV in the gas phase ${ }^{4}$ ) this is not surprising. All these peaks are bleached on $313 \mathrm{~nm}$ photolysis (lower spectrum c).

Fig. 4 juxtaposes the positive peaks in the top spectra (b) and (c) in Fig. 3 to spectra simulated on the basis of B3LYP/ 6-311G(2d,p) calculations. The good accord leaves little doubt that the above-proposed assignments to the radical ions of $\mathrm{PBQ}$ are correct. An assignment of the IR spectra of $\mathrm{PBQ}^{\bullet-}$ and $\mathrm{PBQ}^{\bullet+}$ will be given in the theoretical section.

The reader will have noticed that we have refrained from including the section above $1800 \mathrm{~cm}^{-1}$ in our analysis of the IR spectra. The reason is that this region contains not only the expected vibrational transitions due to $\mathrm{C}-\mathrm{H}$ stretching vibrations in the $2900-3100 \mathrm{~cm}^{-1}$ range, but in addition many other very prominent features which are shown in Fig. 5. As most of these features are absent when DABCO is present, they must be due to the radical cation of $\mathrm{PBQ}$, which is not entirely unexpected in view of the close proximity of the first excited state and the

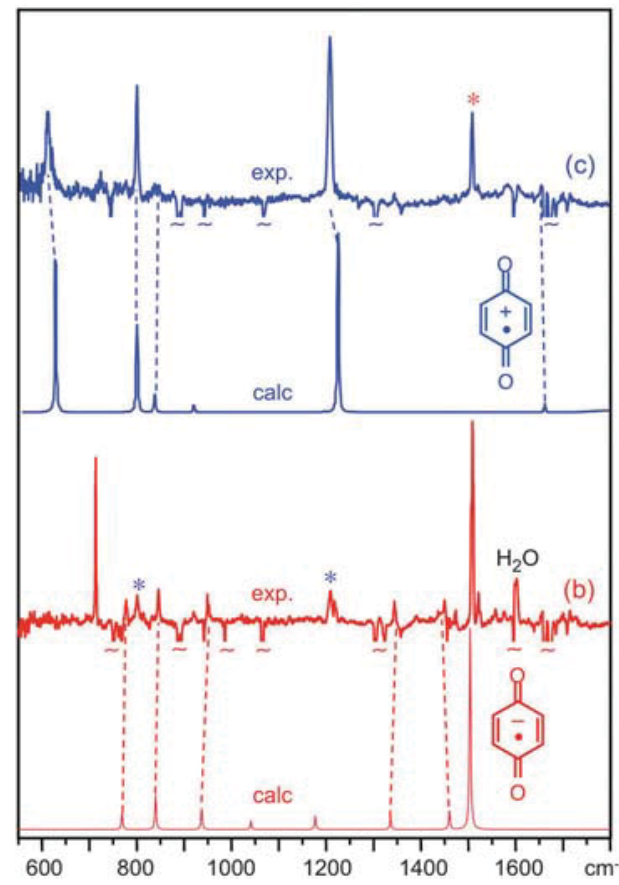

Fig. 4 Spectra (b) and (c) of Fig. 3, juxtaposed to spectra simulated on the basis of B3LYP/6-311G(2d,p) calculations (frequencies scaled by 0.976 ). Asterisks denote peaks of $\mathrm{PBQ}^{\bullet-}$ in the spectrum of $\mathrm{PBQ}^{\bullet}{ }^{+}$, and vice versa.

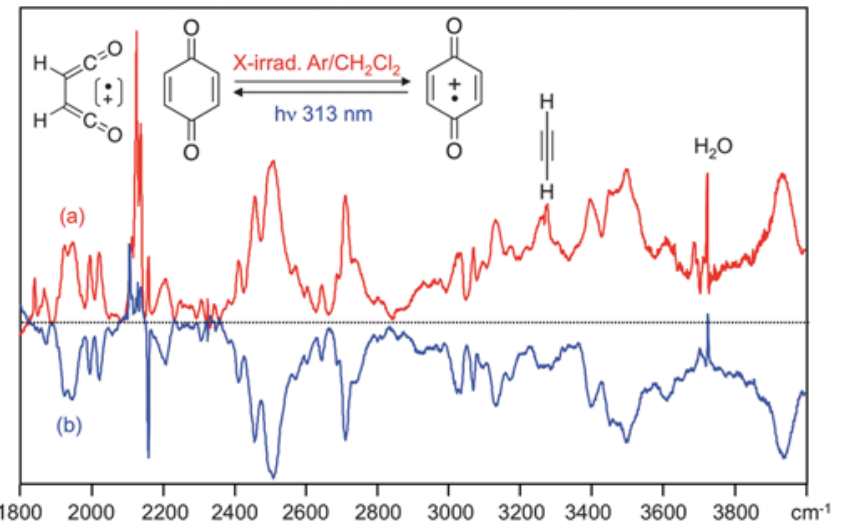

Fig. 5 Near-IR difference spectrum for the X-irradiation of an Ar matrix containing $P B Q$ and $\mathrm{CH}_{2} \mathrm{Cl}_{2}$ (a), and for the subsequent bleaching at $313 \mathrm{~nm}$ (b).

ground state that had been revealed by the analysis of the photoelectron spectrum. ${ }^{23}$

Before going into an analysis of what appears to be the first electronic transition of $\mathrm{PBQ}^{\bullet+}$, we look at some features that arise from other carriers, because they are not (or only partially) bleachable by $313 \mathrm{~nm}$ photolysis. First among those are the sharp peaks at $2100-2200 \mathrm{~cm}^{-1}$ which can be assigned quite unambiguously to bisketene and its radical cation, which we have characterized independently by way of ionization of cyclobutenedione. ${ }^{27}$ Upon $313 \mathrm{~nm}$ photolysis, the radical cation of bisketene is transformed into the neutral species. As bisketene must arise by fragmentation of $\mathrm{PBQ}^{+} \bullet$, it must be accompanied by acetylene, which is characterized by the asymmetric $\mathrm{C}-\mathrm{H}$ stretching vibration at $3277 \mathrm{~cm}^{-1}$ (actually a doublet, due to a 
well-known Darling-Dennison resonance), another feature that is not bleached on $313 \mathrm{~nm}$ photolysis. Finally, the sharp peaks at around $3725 \mathrm{~cm}^{-1}$ are due to matrix-isolated water.

The remaining, more broad features must be due to the $\mathrm{X}^{2} \mathrm{~B}_{3 \mathrm{~g}} \rightarrow \mathrm{A}^{2} \mathrm{~B}_{2 \mathrm{u}}$ electronic transition of $\mathrm{PBQ}^{+\bullet}$. The unusual and complicated shape of this electronic transition is doubtlessly due to the vibronic coupling between these two states, ${ }^{23}$ which will be addressed below.

\section{Theoretical discussion}

Our discussion of the vibrational structure given below will be based on the choice of axes shown in Fig. 1, and on Herzberg's convention for numbering frequencies. ${ }^{28}$

\subsection{Vibrational structure of PBQ}

In $D_{2 \mathrm{~h}}$ symmetry, the 30 fundamentals of PBQ are divided into 15 Raman- and 13 IR-active modes (the remaining two being inactive $\mathrm{a}_{\mathrm{u}}$ fundamentals). Of the expected IR bands, two are $\mathrm{C}-\mathrm{H}$ stretches, so that 11 fundamentals should be observable below $2000 \mathrm{~cm}^{-1}$. According to a scaled quantum chemical force field calculation ${ }^{29}$ three of these lie below $500 \mathrm{~cm}^{-1}$, i.e. outside our observation range. Of the eight remaining fundamentals, all but the weakest one (predicted at $c a .733 \mathrm{~cm}^{-1}$ with an intensity of $0.3 \mathrm{~km} \mathrm{~mol}^{-1}$, based on DFT calculations) were identified in the matrix spectrum shown in Fig. $3 \mathrm{a}$.
This spectrum is in excellent accord with previous measurements in the gas and in condensed phases. ${ }^{26,30,31}$ The assignment of this spectrum, in particular that of the pair of intense bands at $1650-1670 \mathrm{~cm}^{-1}$, which appear prominently in the matrix spectrum, had been controversial, because calculations predict only one fundamental in this region. However, it was established that the two bands which are peaking at 1659 and $1671 \mathrm{~cm}^{-1}$ in our matrix spectra are due to two Fermi-coupled $\mathrm{b}_{1 \mathrm{u}}$ vibrational states. ${ }^{32}$ One is the antisymmetric $\mathrm{C}=\mathrm{O}$ stretching fundamental and the other is the combination of $a b_{2 u}$ fundamental, which peaks at $1067 \mathrm{~cm}^{-1}$ in the matrix spectrum, and a $b_{3 g}$ fundamental which is observed at $c a .600 \mathrm{~cm}^{-1}$ in the Raman spectrum of PBQ. ${ }^{33,34}$ Which is which has been a matter of some debate, but the states are likely to be so strongly mixed as to render the question moot. In addition we observe a few weak bands at higher energies which we assign to combination bands in accord with Charney and Becker $^{26}$ ( $c f$. Table 1).

A feature of the IR-spectrum of PBQ that we would like to retain for future reference is that the most intense fundamental transition in the spectrum, the antisymmetric $\mathrm{C}=\mathrm{O}$ stretch, has an intensity of $c a .300 \mathrm{~km} \mathrm{~mol}^{-1}$, according to our B3LYP calculations.

\subsection{Electronic and vibrational structure of $\mathrm{PBQ}^{\bullet}$}

The electronic structure of $\mathrm{PBQ}^{\bullet-}$ has been at the focus of several theoretical studies, all of which agree that the ground state of this

Table 1 Assignments of the observed absorption peaks of PBQ and its radical cation and anion observed in the matrix isolation spectroscopic measurements and predicted by B3LYP/6-311G (2d,p) calculations (all frequencies scaled by 0.976)

\begin{tabular}{|c|c|c|c|c|}
\hline \multicolumn{2}{|l|}{ Peak position $\left(\mathrm{cm}^{-1}\right)$} & \multicolumn{3}{|l|}{ Assignments } \\
\hline $\begin{array}{l}\text { B3LYP (intensity), } \\
\text { italic: combination bands }\end{array}$ & Experimental & $\mathrm{PBQ}^{+\bullet} \mathrm{X}^{2} \mathrm{~B}_{3 \mathrm{~g}}$ & $\mathrm{PBQ}^{-\bullet} \mathrm{X}^{2} \mathrm{~B}_{2 \mathrm{~g}}$ & PBQ $X{ }^{1} A_{g}$ \\
\hline $631 \quad(875)$ & $613 \quad(\mathrm{~s})$ & $14^{1}\left(b_{1 u}\right)$ & & \\
\hline $769 \quad(27)$ & $778 \quad(w)$ & & $14^{1}\left(\mathrm{~b}_{1 \mathrm{u}}\right)$ & \\
\hline $805 \quad(591)$ & $801 \quad(\mathrm{~s})$ & $13^{1}\left(b_{1 u}\right)$ & & \\
\hline $840 \quad(54)$ & $848 \quad(w)$ & & $28^{1}\left(b_{3 u}\right)$ & \\
\hline 851 & $846 \quad$ (w) & $28^{1}\left(b_{3 u}\right)$ & & \\
\hline $893 \quad(73)$ & $888 \quad(\mathrm{~s})$ & & & $28^{1}\left(b_{3 u}\right)$ \\
\hline 929 (19) & $942 \quad(\mathrm{~m})$ & & & $13^{1}\left(b_{1 u}\right)$ \\
\hline (34) & $949 \quad(w)$ & & $13^{1}\left(b_{1 u}\right)$ & \\
\hline $930 \quad(38)$ & 952 (w) & $21^{1}\left(b_{2 u}\right)$ & & \\
\hline $1042 \quad(20)$ & ? & & $21^{1}\left(b_{2 u}\right)$ & \\
\hline 1056 & $1067 \quad(\mathrm{~m})$ & & & $21^{1}\left(b_{2 u}\right)$ \\
\hline 1178 & $\approx 1210$ & & $20^{1}\left(b_{2 u}\right)$ & \\
\hline $1247 \quad(1041)$ & 1209 (vs) & $12^{1}\left(b_{1 u}\right)$ & & \\
\hline 1282 & 1303 (s) & & & $20^{1}\left(\mathrm{~b}_{2 \mathrm{u}}\right)$ \\
\hline 1320 & 1332 (w) & & & $14^{1} 26^{1}\left(b_{1 u} \otimes b_{3 g}\right)$ \\
\hline 1337 & 1344 (w) & & $12^{1}\left(b_{1 u}\right)$ & \\
\hline 1352 & 1358 (w) & & & $12^{1}\left(b_{1 u}\right)$ \\
\hline 1462 & $1450 \quad(w)$ & & $19^{1}\left(\mathrm{~b}_{2 \mathrm{u}}\right) ; \nu^{\mathrm{as}} \mathrm{C}=\mathrm{C}$ & \\
\hline 1433 & 1474 (w) & & $15^{1} 29^{1}\left(b_{2 g} \otimes b_{3 u}\right)$ & \\
\hline$(327)$ & 1509 (vs) & & $11^{1}\left(\mathrm{~b}_{1 \mathrm{u}}\right) ; \nu^{\mathrm{as}} \mathrm{C}=\mathrm{O}$ & \\
\hline 1503 & 1522 (w) & & $21^{1} 27^{1}\left(b_{2 u} \otimes b_{3 g}\right)$ & \\
\hline 1566 & 1558 (w) & & $5^{1} 14^{1}\left(a_{g} \otimes b_{1 u}\right)$ & \\
\hline 1589 & $1580 \quad(w)$ & & $16^{1} 28^{1}\left(b_{2 g} \otimes b_{3 u}\right)$ & \\
\hline 1602 & 1596 (m) & & & $19^{1}\left(\mathrm{~b}_{2 \mathrm{u}}\right) ; \nu^{\text {as }} \mathrm{C}=\mathrm{C}$ \\
\hline 1668 & 1655 (w) & $11^{1}\left(\mathrm{~b}_{1 \mathrm{u}}\right) \nu^{\mathrm{as}} \mathrm{C}=\mathrm{O}$ & & \\
\hline$(354)$ & 1659 (vs) & & & $11^{1}\left(\mathrm{~b}_{1 \mathrm{u}}\right) ; \nu^{\mathrm{as}} \mathrm{C}=\mathrm{O}$ \\
\hline 1643 & 1671 (vs) & & & $21^{1} 26^{1}\left(b_{2 u} \otimes b_{3 g}\right)$ \\
\hline 1692 & $1684 \quad(\mathrm{~m})$ & & & $16^{1} 28^{1}\left(b_{2 g} \otimes b_{3 u}\right)$ \\
\hline 1680 & 1709 (w) & & & $5^{1} 13^{1}\left(a_{g} \otimes b_{1 u}\right)$ \\
\hline
\end{tabular}


anion may be regarded as arising by addition of an electron to the $b_{2 g}$ LUMO of PBQ ( $c f$. Fig. 1), and that the excited states observed in the electronic absorption spectra are not the lowest ones. ${ }^{19-21}$ However, which states contribute in what order to the observed features has not been settled unambiguously: CASSCF/ CASPT2 calculations predict two very close-lying doublet states of $\mathrm{B}_{3 \mathrm{u}}$ and $\mathrm{A}_{\mathrm{u}}$ symmetry, respectively, near $2.8 \mathrm{eV}(440 \mathrm{~nm})$ and a more intense transition to another ${ }^{2} \mathrm{~B}_{3 \mathrm{u}}$ state at $3.56 \mathrm{eV}(350 \mathrm{~nm})^{21}$ (in addition to two "silent" states at $c a .2 .25 \mathrm{eV}$ ). From calculated vibrational profiles the authors of that study concluded that the vibronic structure of the band peaking at $450 \mathrm{~nm}$ indicates that it is due to ${ }^{2} \mathrm{~B}_{2 \mathrm{~g}} \rightarrow{ }^{2} \mathrm{~B}_{3 \mathrm{u}}$ transition, whereas the transition to the ${ }^{2} \mathrm{~A}_{\mathrm{u}}$ state should give rise to a broad band. This implies that the band at $400-500 \mathrm{~nm}$ comprises excitations to two states, whereas that peaking at $320 \mathrm{~nm}$ corresponds to the second ${ }^{2} \mathrm{~B}_{2 \mathrm{~g}} \rightarrow{ }^{2} \mathrm{~B}_{3 \mathrm{u}}$ transition.

In contrast, the SAC-CI study of Honda et al.,${ }^{19}$ which predicts eleven "silent" states below $2.6 \mathrm{eV}(475 \mathrm{~nm})$ places the ${ }^{2} \mathrm{~A}_{\mathrm{u}}$ state at $2.71 \mathrm{eV}(458 \mathrm{~nm})$, and the two ${ }^{2} \mathrm{~B}_{3 \mathrm{u}}$ states at 3.5 and $3.74 \mathrm{eV}$, respectively ( 355 and $330 \mathrm{~nm}$ ) which leads to the conclusion that the first band corresponds to a single electronic transition, whereas the intense UV-band comprises two transitions.

In this context we wish to point out the presence of a weak feature to the red of the leading $450 \mathrm{~nm}$ peak in the spectra shown in Fig. 2, which is reproducible and not due to an impurity. The same feature appears, better resolved, in the matrix spectrum of Gebicki and Michl $^{10}$ (which was ignored by the above-cited theoretical studies). We propose that this feature indicates the presence of a second excited state in this region, perhaps the ${ }^{2} \mathrm{~A}_{\mathrm{u}}$ state that was predicted in this region by CASSCF/CASPT2. Our own TD-DFT calculations (see ESI $\dagger$ ) are in agreement with that assignment, and they also predict a single excited state in the $320 \mathrm{~nm}$ region. However, at this point, the interpretation of the electronic spectrum of $\mathrm{PBQ}^{\bullet-}$ remains speculative, and a topic that should be further investigated.

The vibrational structure of $\mathrm{PBQ}^{\bullet-}$ has stood at the focus of several studies, most of which have probed the totally symmetric fundamentals by (resonance) Raman spectroscopy. ${ }^{11-14}$ In the IR spectrum only the most intense band at around $1500 \mathrm{~cm}^{-1}$ had been unambiguously pinpointed. ${ }^{14,16}$ The vibrational structure of $\mathrm{PBQ}^{--}$has been investigated computationally by different groups, ${ }^{35,36}$ but since they had done this in view of assigning the Raman spectra, they did not list the predicted IR intensities. The spectrum simulated on the basis of B3LYP calculations being in excellent accord with the pattern of observed bands indicates that these calculations may serve as an adequate basis for an assignment of the experimental spectrum.

According to these calculations, three of the 13 IR-active fundamentals are expected below $500 \mathrm{~cm}^{-1}$ and two are $\mathrm{C}-\mathrm{H}$ stretches which leaves eight others in the region plotted in Fig. 4, six of which can be assigned quite unambiguously from our matrix spectra.

The most intense band is, as in neutral PBQ, due to the previously observed asymmetric $\mathrm{C}=\mathrm{O}$ stretching fundamental of $b_{1 u}$ symmetry, which, however, is evidently not subject to such strong Fermi coupling to a combination band in the anion as it is in the neutral species. Its frequency decreases from $1659 \mathrm{~cm}^{-1}$ in PBQ to $1509 \mathrm{~cm}^{-1}$, which is due to the weakening of the $\mathrm{C}=\mathrm{O}$ bonds upon adding an electron to the LUMO ( $c f$. Fig. 1). The intensity of the band is comparable to that of the neutral species.

The antisymmetric $\mathrm{C}=\mathrm{C}$ stretching vibration, which is at $1596 \mathrm{~cm}^{-1}$ in the neutral species, appears at $1450 \mathrm{~cm}^{-1}$ in the anion, a shift which can again be explained by the $\mathrm{C}=\mathrm{C}$ antibonding nature of the LUMO of PBQ. This band is followed by peaks at $1344,949,848$, and $778 \mathrm{~cm}^{-1}$ that are assigned to $\mathrm{C}-\mathrm{H}$ wagging and ring deformation vibrations. Two $b_{2 u}$ fundamentals, $\nu_{20}$ and $\nu_{21}$, predicted at 1178 and $1042 \mathrm{~cm}^{-1}$, respectively, with similar intensities as other observed bands, cannot be clearly discerned in the experimental spectrum (perhaps the former is hidden underneath the very intense $\nu_{12}\left(\mathrm{~b}_{1 \mathrm{u}}\right)$ of $\mathrm{PBQ}^{{ }^{+}}$at $1209 \mathrm{~cm}^{-1}$ ). The relatively intense band at $1522 \mathrm{~cm}^{-1}$ might be due to a combination band $\left(b_{2 u} \otimes b_{3 g}\right.$, predicted at $1503 \mathrm{~cm}^{-1}$ by the scaled B3LYP calculations) which gains some intensity through Fermi coupling to the nearby and strong $b_{1 u}$ fundamental. The weak bands that appear at 1474, 1558 and $1580 \mathrm{~cm}^{-1}$ are also tentatively assigned in terms of combinations of $\mathrm{g}$ and $\mathrm{u}$ vibrations predicted nearby.

\subsection{Electronic and vibrational structure of $\mathrm{PBQ}^{\circ+}$}

Apart from the very unusual shape of the electronic absorption band shown in Fig. 5, which will be discussed separately below, the most striking spectroscopic features of $\mathrm{PBQ}^{\bullet+}$ are the pronounced intensities of the three major peaks, at 613, 801, and $1209 \mathrm{~cm}^{-1}$, in the IR-spectrum ( $c f$. Fig. 4), assigned in each case to fundamental transitions that have either modest intensity or are not observed at all in the neutral and anionic states of PBQ. As assigned by B3LYP calculations, these three transitions involve vibrations of $\mathrm{b}_{1 \mathrm{u}}$ symmetry and have intensities that are also significantly higher than that of the most intense IR band of the neutral species or the radical anion, i.e. the asymmetric $\mathrm{C}=\mathrm{O}$ stretch, which in $\mathrm{PBQ}^{\bullet+}$ is actually predicted to be much weaker than in the neutral species or the anion (see Fig. 6).

The large intensities of the IR-absorptions at 613, 801 and $1209 \mathrm{~cm}^{-1}$ arise because vibrations of $b_{1 u}$ symmetry are those which couple the ${ }^{2} \mathrm{~B}_{3 \mathrm{~g}}$ ground state of $\mathrm{PBQ}^{\bullet+}$ to its low-lying ${ }^{2} \mathrm{~B}_{2 \mathrm{u}}$ excited state. ${ }^{37,38}$ By doing so the vibrations decrease in frequency and the corresponding IR-transitions "borrow intensity" from the $\mathrm{X}^{2} \mathrm{~B}_{3 \mathrm{~g}} \rightarrow \mathrm{A}^{2} \mathrm{~B}_{2 \mathrm{u}}$ electronic transition. Although DFT may not be the optimal method to treat nonadiabatic effects, ${ }^{39,40}$ we note that these effects express themselves already quite clearly at this level, as illustrated in Fig. 6 which shows the four $\mathrm{b}_{1 \mathrm{u}}$ normal modes of PBQ (the fifth one is a C-H stretch) and its radical cation, as well as the (unscaled) frequencies and intensities of the IR transitions. In three of them, mixtures of $\mathrm{C}-\mathrm{H}$ wagging and ring deformation modes, the frequencies decrease indeed (by 105-127 $\mathrm{cm}^{-1}$ ) while the intensities dramatically increase (from $0.5-18.5$ to $600-1000 \mathrm{~km} \mathrm{~mol}^{-1}$ ). In contrast, the frequency of the antisymmetric $\mathrm{C}=\mathrm{O}$ stretching vibration is predicted to decrease by only $25 \mathrm{~cm}^{-1}$ while its intensity decreases actually by a factor of over 4 (the tentatively assigned 

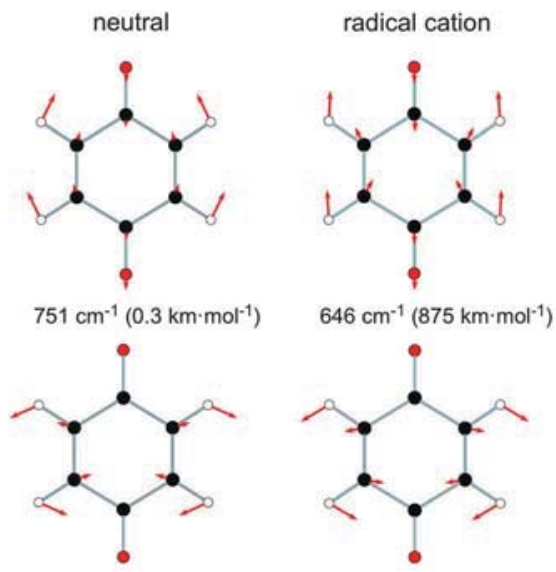

$952 \mathrm{~cm}^{-1}\left(18.8 \mathrm{~km} \cdot \mathrm{mol}^{-1}\right)$

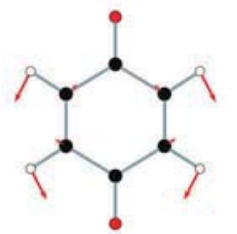

$1385 \mathrm{~cm}^{-1}\left(5.8 \mathrm{~km} \cdot \mathrm{mol}^{-1}\right)$
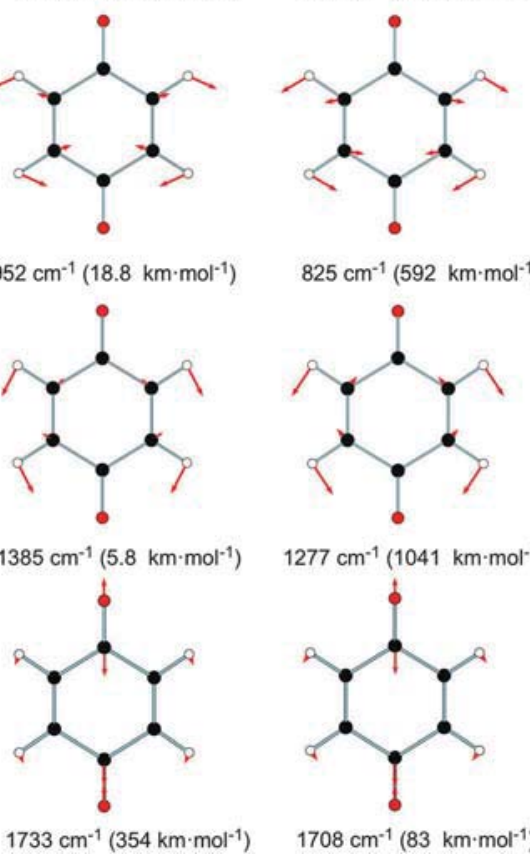

$825 \mathrm{~cm}^{-1}\left(592 \mathrm{~km} \cdot \mathrm{mol}^{-1}\right)$

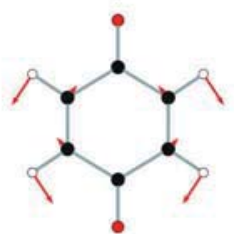

$1277 \mathrm{~cm}^{-1}\left(1041 \mathrm{~km} \cdot \mathrm{mol}^{-1}\right)$

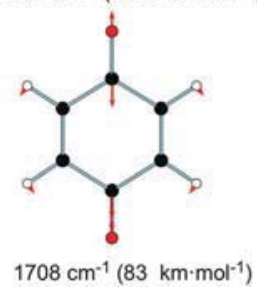

Fig. $6 b_{1 u}$ Vibrations of PBQ (left) and its radical cation (right). Wavenumbers and intensities (both unscaled) are from B3LYP/6-311G(2d,p) calculations.

weak experimental feature is barely shifted from that of the neutral state).

How effective different vibrations of the proper symmetry are at coupling two states depends on the projection of the corresponding normal modes onto the diabatic coupling vector between the two electronic states involved (i.e. the distortion that optimally couples the two states).$^{38,41}$ These vectors, which are needed to find conical intersections between two states, are often computed by the CASSCF method whereby the orbitals are averaged over the two coupled states. In the case of $\mathrm{PBQ}^{\bullet+}$ such a calculation yields a vector that is strongly localized along the antisymmetric $\mathrm{C}=\mathrm{O}$ stretching mode, i.e. the mode which appears to be least affected by vibronic coupling.

However, Fig. 7 shows that, if one averages the orbitals over three or four states, respectively, other modes begin to participate increasingly in the derivative coupling vector. Finally, equation-of-motion coupled cluster theory (EOMIP-CCSD) predicts a coupling vector that is quite delocalized amongst the modes of $b_{1 u}$ symmetry, in accord with what the IR spectrum expresses. We have no explanation for this failure of a CASSCF calculation where orbitals are only averaged over the two coupling states to reproduce this result.

A simple perturbation analysis, outlined in the Appendix, shows that the EOMIP-CCSD coupling calculation is entirely

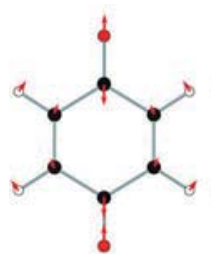

(a)

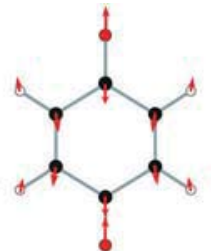

(b)

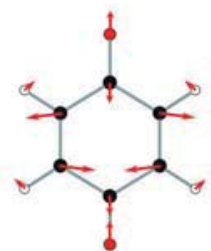

(c)

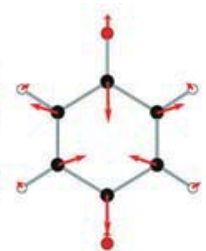

(d)
Fig. 7 Derivative coupling vectors from CASSCF calculations where the orbitals are averaged over (a) 2 states, (b) 3 states, and (c) 4 states, and from EOMIP-CCSD calculations for $\mathrm{PBQ}^{\bullet+}$ (d).

consistent with the infrared spectrum provided by the same method (which is qualitatively the same as that obtained by the B3LYP method). In particular, the fact that the intensity of the antisymmetric $\mathrm{C}=\mathrm{O}$ stretch is smaller in the cation than in the neutral species and that its frequency is largely unaffected can be understood by this analysis which shows that the vibronic contributions to the vibrational intensities (i.e. the contributions that are a direct result of the electronic state coupling) are the overwhelmingly dominant part of the transition moment for the very strong low-frequency features. However, for the antisymmetric $\mathrm{C}=\mathrm{O}$ stretch, the intrinsic transition moment and the vibronic contribution have different phases, which results in their destructive interference and a net reduction of the intensity of that transition in the radical cation.

With regard to the frequency, we note that the $\mathrm{C}=\mathrm{O}$ distance in the cation is $c a .0 .02 \AA$ shorter than in the neutral species; thus, in the absence of vibronic interactions, one would expect the corresponding stretching frequency in the cation to be higher than in the neutral species. Consequently, the small change in the frequency between neutral species and the cation comprises a positive contribution to the intrinsic frequency from bond compression and a negative contribution from the vibronic interaction, a cancellation of effects that is qualitatively similar to that seen in the transition moment itself.

Obviously, the strong vibronic coupling between these two states affects also the electronic transition between them. Consequently, the Born-Oppenheimer approximation breaks down and one cannot model that transition on the basis of FranckCondon factors calculated for separate potential surfaces of the two states.

The envelope of the first band of the electronic spectrum, which manifests itself vividly in the region above $1800 \mathrm{~cm}^{-1}$, is extremely complicated, as both states that are involved in the corresponding transition are admixtures of the diabatic ${ }^{2} \mathrm{~B}_{3 \mathrm{~g}}$ and the ${ }^{2} \mathrm{~B}_{2 \mathrm{u}}$ electronic states. As in our earlier photoelectron spectroscopic study, ${ }^{23}$ a quasidiabatic model Hamiltonian method $^{41}$ turns out to be useful to model the properties of the nonadiabatic system.

The results of the simulation of the first electronic absorption band based on a quadratic vibronic coupling hamiltonian are shown in Fig. 8. The agreement with experiment can only be said to be qualitative, but many of the features seen in the experiment are also found in the simulated spectrum. While details of the spectral analysis will be given in a separate publication, ${ }^{42}$ 


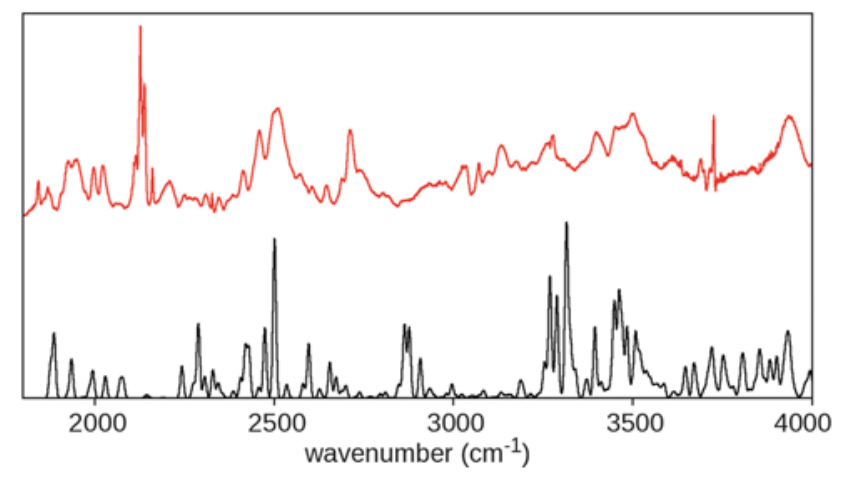

Fig. 8 IR difference spectrum from Fig. 5 (top) and a simulated spectrum of the $\mathrm{A}^{2} \mathrm{~B}_{2 \mathrm{u}} \leftarrow \mathrm{X}^{2} \mathrm{~B}_{3 \mathrm{~g}}$ electronic transition of $\mathrm{PBQ}^{\bullet+}$ (bottom).

the simulation supports the idea that a low-lying electronic transition in this system, complicated by strong vibronic coupling effects, is the underlying reason for the unusual appearance of the band envelope in this region of the spectrum.

\section{Conclusions}

We have generated the radical ions (anion and cation) of p-benzoquinone (PBQ) by X-irradiation of neutral $\mathrm{PBQ}$ in an Ar matrix at $10 \mathrm{~K}$ and recorded their UV-Vis and the IR absorption spectra. The matrix IR spectrum of PBQ is in excellent accord with previous measurements in the gas and in condensed phases, and shows a prominent Fermi resonance splitting of the intense antisymmetric $\mathrm{C}=\mathrm{O}$ stretch.

Our electronic absorption spectrum of $\mathrm{PBQ}^{\bullet-}$ supports the notion that the first band system at $350-500 \mathrm{~nm}$ comprises transitions to two states while the second band which peaks at $320 \mathrm{~nm}$ contains only one, in accord with CASSCF/CASPT2 and TDDFT but in contradiction with SAC-CI calculations. In the vibrational spectrum of $\mathrm{PBQ}^{\bullet-}$ six of the eight IR-active fundamentals between 500 and $2000 \mathrm{~cm}^{-1}$ were observed and assigned.

The spectra of the radical cation of $\mathrm{PBQ}$, which are reported for the first time in this paper, are remarkable in several respects: the lowest energy band in the electronic absorption spectrum (which lies fully in the mid-IR region between 1900 and $4500 \mathrm{~cm}^{-1}$ ) shows a very complicated envelope; the vibrational spectrum below $1700 \mathrm{~cm}^{-1}$ is dominated by three exceptionally strong peaks (calculated intensities of $590-1040 \mathrm{~km} \mathrm{~mol}^{-1}$ ) below $1300 \mathrm{~cm}^{-1}$; conversely, the infrared-active antisymmetric $\mathrm{C}=\mathrm{O}$ stretch, which is by far the most intense band in neutral PBQ and its radical anion, is barely visible in the $\mathrm{PBQ}^{\bullet+}$ spectrum.

All three unusual features noted above can be rationalized by recognizing that the ${ }^{2} \mathrm{~B}_{3 \mathrm{~g}}$ ground state and the low-lying ${ }^{2} \mathrm{~B}_{2 \mathrm{u}}$ excited state undergo pronounced coupling through vibrations of $b_{1 u}$ symmetry. As a result, it is not appropriate to model the electronic transition between these two mixed states by means of a Franck-Condon analysis between the respective adiabatic potential energy surfaces. Moreover, the four IR-active $b_{1 u}$ vibrations "borrow" intensity from the electronic transition which explains the prominence that they exhibit in the IR-spectrum. The $\mathrm{C}=\mathrm{O}$ stretch does not profit from this intensity enhancement because for this mode the intrinsic transition moment and the vibronic contribution have different phases, which leads to their destructive interference and a greatly diminished intensity relative to the same mode in the neutral species and the anion.

\section{Methods}

\section{Compounds}

$p$-Benzoquinone (PBQ) and diaza[2.2.2]bicyclooctane (DABCO) were used as obtained in high purity form.

\section{Matrix isolation and ionization}

PBQ was mixed in a 1:1000 ratio with a 9:1 mixture of Ar and $\mathrm{N}_{2}$ (where the latter is added to improve the optical quality of the matrices) in a $2000 \mathrm{ml}$ storage vessel to a total pressure of 100 mbar, about half of which was deposited over $c a$. an hour on a CsI window held at $20 \mathrm{~K}$ inside the cold head of a closedcycle cryostat fitted with $\mathrm{KBr}$ windows. In experiments with $\mathrm{DABCO}$, this was premixed with $\mathrm{PBQ}$ in a $1: 1$ ratio before adding the gas mixture. After completing the deposition, the heater of the cryostat was switched off, so the temperature of the matrix fell to $10 \mathrm{~K}$. X-irradiation was effected by means of a source tube containing a tungsten anode that was operated at $40 \mathrm{kV} / 40 \mathrm{~mA}$, which gives off Bremsstrahlung that under these conditions peaks at $c a$. $0.5 \AA / 25 \mathrm{keV}$. Under the impact of this radiation, electron-hole pairs form in the Ar matrix, a few of which separate and diffuse (in the form of polarons) through the solid until they are trapped by added substrates or impurities with a lower oxidation or reduction potential, respectively, than Ar. In the absence of DABCO mainly the radical cation of $\mathrm{PBQ}$ forms in this process, while the electrons that are not trapped by PBQ are probably trapped by impurities in the matrix. When one adds DABCO, most of the holes are trapped by this easily oxidized compound, thus largely suppressing the formation of $\mathrm{PBQ}^{\bullet+}$, while most of the electrons continue to be trapped by PBQ and the spectrum of its radical anion becomes more prominent.

\section{Apparatus}

UV/VIS/NIR spectra were recorded on a Perkin-Elmer Lambda 900 instrument, while IR spectra were obtained on a Bomem DA3 interferometer equipped with a $\mathrm{KBr}$ beamsplitter and a MCT detector. Usually, 256 interferograms taken at $0.5 \mathrm{~cm}^{-1}$ resolution were added before Fourier transformation of each spectrum.

\section{Quantum chemical calculations}

Geometries were optimized and frequencies were calculated by the (U)B3LYP/6-311G(2d,p) method using the Gaussian 09 program system. ${ }^{43}$ Additionally, model Hamiltonian calculations of the spectrum were based on a quasidiabatic Hamiltonian parametrized on the basis of calculations using the equation-of-motion coupled cluster method known as EOMIPCCSD,${ }^{44}$ using a double-zeta plus polarization basis set. The vibronic calculation used the "adiabatic parametrization" discussed in ref. 45 with coupling constants calculated using the analytic scheme presented in $2009 .{ }^{46}$ 


\section{Appendix}

Table 2 shows the results of the EOMIP-CCSD calculations regarding the $b_{1 \mathrm{u}}$ fundamentals in the ${ }^{2} \mathrm{~B}_{3 \mathrm{~g}}$ ground state of $\mathrm{PBQ}^{\bullet+}$. The rightmost two columns are the actual EOMIP-CCSD results obtained with a DZP basis set, while the remaining numbers are obtained with the simple qualitative model for vibronic coupling described below.

At the equilibrium geometry of the neutral state, the ${ }^{2} \mathrm{~B}_{2 \mathrm{u}}$ excited state lies $3400 \mathrm{~cm}^{-1}$ above the ground state at the EOMIP-CCSD level, and the diabatic coupling vector calculated (in the dimensionless normal coordinates of the radical cation ground state) are: $996\left(\nu_{10}\right),-397\left(\nu_{11}\right),-483\left(\nu_{12}\right),-284\left(\nu_{13}\right)$, and $167\left(\nu_{14}\right) \mathrm{cm}^{-1}$. A qualitatively useful and simple treatment of the vibronic interaction in which only the ground and first-excited vibrational states of each electronic state are considered, and each fundamental vibration is treated as separable, is provided by projecting the linear vibronic coupling Hamiltonian ${ }^{41}$

$$
H=\left(\begin{array}{cc}
\frac{1}{2} \omega q^{2} & \lambda q \\
\lambda q & \Delta+\frac{1}{2} \omega q^{2}
\end{array}\right)
$$

(where $\omega$ is the unperturbed harmonic frequency of the mode, taken here as that of the corresponding mode in the neutral species, $\Delta$ is the vertical spacing between the electronic states, and $\lambda$ is the coupling strength) onto a simple vibrational basis set consisting of just the zero-quantum (ground) and one-quantum (fundamental) harmonic oscillator functions. Using the lowest order of perturbation theory, this very simple model gives the following vibronic wavefunctions for the ground and singly-excited vibrational states

$$
\begin{aligned}
& \psi_{0}=\Psi_{\mathrm{X}} \phi_{0}-\frac{\lambda}{\sqrt{2}(\Delta+\omega)} \Psi_{\mathrm{A}} \phi_{1} \\
& \psi_{1}=\Psi_{\mathrm{X}} \phi_{1}-\frac{\lambda}{\sqrt{2}(\Delta-\omega)} \Psi_{\mathrm{A}} \phi_{0}
\end{aligned}
$$

where the uppercase wavefunctions represent the unperturbed electronic states, and $\phi_{0}$ and $\phi_{1}$ are the $\nu=0$ and $\nu=1$ harmonic oscillator wavefunctions. The transition moment between these states (to first-order in the coupling strength) is given by

$$
M \equiv\left\langle\psi_{0}|\mu| \psi_{1}\right\rangle=M_{0}+M_{1}+M_{2}
$$

Table 2 Calculated parameters for the $b_{1 u}$ fundamental modes of the PBQ cation. The rightmost columns are results of EOMIP-CCSD calculations with the DZP basis set; the remaining entries are obtained from the

\begin{tabular}{|c|c|c|c|c|c|c|}
\hline & \multicolumn{4}{|c|}{ Model results } & \multicolumn{2}{|c|}{ Cation (EOMIP-CCSD) } \\
\hline & $\omega$ & $M_{0}$ & $M_{1}$ & $M_{2}$ & $\omega_{\mathrm{EOM}}$ & $M_{\mathrm{EOM}}$ \\
\hline$\nu_{10}$ & 3225 & 0.004 & $a$ & -0.030 & 3232 & -0.057 \\
\hline$\nu_{11}$ & 1791 & -0.104 & 0.204 & 0.066 & 1744 & 0.068 \\
\hline$\nu_{12}$ & 1392 & 0.015 & 0.267 & 0.124 & 1243 & 0.280 \\
\hline$\nu_{13}$ & 956 & -0.025 & 0.182 & 0.113 & 810 & 0.229 \\
\hline$\nu_{14}$ & 761 & -0.003 & -0.420 & -0.299 & 568 & -0.530 \\
\hline
\end{tabular}
simple model described here, with parameters obtained from EOMIPCCSD calculations. The quantities $M_{i}$ listed below refer to transition dipole contributions in atomic units (see text)

${ }^{a}$ Perturbation theory is inappropriate here because of a small denominator. where

$$
\begin{gathered}
M_{0} \equiv\left\langle\phi_{0}|\mu| \phi_{1}\right\rangle=\frac{1}{\sqrt{2}}\left(\frac{\partial \mu}{\partial q}\right)_{0} \\
M_{1} \equiv \frac{-\lambda}{\sqrt{2}(\Delta-\omega)} M_{\mathrm{XA}} \\
M_{2} \equiv \frac{-\lambda}{\sqrt{2}(\Delta+\omega)} M_{\mathrm{XA}}
\end{gathered}
$$

In the above equations, $M_{\mathrm{XA}}$ is the electronic moment between the two unperturbed electronic states. Proceeding with this simple model, we take the first term (the usual dipole derivative term associated with the double harmonic approximation) from the neutral species, and use the EOMIP-CCSD values for the couplings, vertical gap, and the electronic transition moment (1.69 a.u.), which leads to the results in Table 2 (dipole in atomic units).

From Table 2 a few things can be seen. First, this simple treatment tends to overestimate the effects of the vibronic contribution to the transition moments, but the results are qualitatively in line with the direct EOMIP-CCSD calculations. For all of the modes except the carbonyl stretch $\left(\nu_{11}\right)$, the vibronic terms $M_{1}$ and $M_{2}$ dominate the total transition moment. While the vibronic contribution for $\nu_{11}$ is not small by any means, it has a different sign than the intrinsic moment (that from the $M_{0}$ contribution). This effectively explains the curious behavior noted for the intensity of this feature in the experimental spectrum. This model is clearly rather crude and qualitative in nature, as it neglects Duschinsky mixing between normal modes, intrinsic shifts in the frequencies associated with geometry changes between the neutral species and the cation (see the text with respect to this issue for $\nu_{11}$ ), and the diagonalization of the vibronic Hamiltonian is grossly approximated here. However, the model has considerable merit for clearly showing the fundamental physics governing the curious behavior of the $b_{1 u}$ modes in the observed infrared spectrum.

\section{Acknowledgements}

This work is part of project No. 2000 20-121747 of the Swiss National Science Foundation. We also thank the US National Science Foundation (Grant CHE-1012743) for financial support.

\section{References}

1 H. Nohl, W. Jordan and R. J. Youngman, Adv. Free Radical Biol. Med., 1986, 2, 211.

2 T.-K. Yang and C.-Y. Shen, 1,4-Benzoquinone, in Encyclopedia of Reagents for Organic Synthesis, ed. L. Paquette, Wiley, New York, 2004, DOI: 10.1002/047084289X.rb033.

3 J. Marks, P. B. Comita and J. I. Brauman, J. Am. Chem. Soc., 1985, 107, 3718.

4 J. Schiedt and R. Weinkauf, J. Chem. Phys., 1999, 110, 304.

5 M. Allan, Chem. Phys., 1983, 81, 235.

6 A. Modelli and P. D. Burrow, J. Phys. Chem., 1984, 88, 3550.

7 T. Shida, Electronic Absorption Spectra of Radical Ions, Elsevier Science Publishing Company, Inc., New York, 1988. 
8 G. N. R. Tripathi, Q. Sun and R. H. Schuler, Chem. Phys. Lett., 1989, 156, 51.

9 A. R. Cook, L. A. Curtiss and J. R. Miller, J. Am. Chem. Soc., 1997, 119, 5729.

10 J. Gebicki and J. Michl, J. Phys. Chem., 1988, 92, 6452.

11 R. E. Hester and K. P. J. Williams, J. Chem. Soc., Faraday Trans. 2, 1982, 78, 573.

12 R. H. Schuler, G. N. R. Tripathi, M. F. Prebenda and D. M. Chipman, J. Phys. Chem., 1983, 87, 5357.

13 S. M. Beck and L. E. Brus, J. Am. Chem. Soc., 1982, 104, 4789.

14 X. J. Zhao, H. Imahori, C.-G. Zhan, Y. Sakata, S. Iwata and T. Kitagawa, J. Phys. Chem. A, 1997, 101, 622.

15 The resonance Raman spectra reported in ref. 8 also exhibit the fundamental levels of some non-totally symmetric modes, presumably as a result of non-adiabatic coupling in the electronically excited states of the radical anion.

16 M. Bauscher and W. Mäntele, J. Phys. Chem., 1992, 96, 11101.

17 P. Ćársky and R. Zahradník, Collect. Czech. Chem. Commun., 1971, 36, 961.

18 H. M. Chang, H. H. Jaffé and C. A. Masmanidis, J. Phys. Chem., 1975, 79, 1118.

19 Y. Honda, M. Hada, M. Ehara and H. Nakatsuji, J. Phys. Chem. A, 2002, 106, 3838.

20 R. A. Wheeler, J. Phys. Chem., 1993, 97, 1533.

21 R. Pou-Amérigo, L. Serrano-Andrés, M. Merchán, E. Ortí and N. Forsberg, J. Am. Chem. Soc., 2000, 122, 6067.

22 L. Klasinc and S. P. McGlynn, in The Chemistry of Quinonoid Compounds, ed. S. Patai and Z. Rappoport, Wiley, New York, vol. 2, 1988.

23 J. F. Stanton, K. W. Sattelmeyer, J. Gauss, M. Allan, T. Skalicky and T. Bally, J. Chem. Phys., 2001, 115, 1.

24 T. Bally, Matrix isolation, in Reactive Intermediate Chemistry, ed. R. A. Moss, M. S. Platz and M. Jones Jr., Wiley, New York, 2004, p. 820.

25 M. Czerwinska, A. Sikora, P. Szajerski, J. Zielonka, J. Adamus, A. Marcinek, K. Piech, P. Bednarek and T. Bally, J. Org. Chem., 2006, 71, 5312.

26 E. Charney and E. D. Becker, J. Chem. Phys., 1965, 42, 910.
27 T. Tidwell, K. Piech and T. Bally, J. Org. Chem., 2013, 78, 2908.

28 G. Herzberg, Molecular Spectra and Molecular Structure, II: Infrared and Raman Spectra of Polyatomic Molecules, Van Nostrand, New York, 1945.

29 R. Liu, X. Zhou and P. Pulay, J. Phys. Chem., 1992, 96, 4255.

30 T. Anno and A. Sado, Bull. Chem. Soc. Jpn., 1958, 31, 734.

31 M. Davies and F. E. Prichard, Trans. Faraday Soc., 1963, 59, 1248.

32 E. D. Becker, H. Ziffer and E. Charney, Spectrochim. Acta, 1963, 19, 1871.

33 H. Stammreich and T. Teixeira Sans, J. Chem. Phys., 1965, 42, 920 .

34 E. D. Becker, J. Phys. Chem., 1991, 95, 2818.

35 C.-G. Zhan and S. Iwata, Chem. Phys., 1998, 230, 45.

36 D. M. Chipman and M. F. Prebenda, J. Phys. Chem., 1986, 90, 5557.

37 According to principles of group theory, vibrations of an irreducible representation that is contained in the direct product of those of a pair of electronic states lead to a symmetry reduction such that the two states have the same symmetry, and can thus interact. In the present case this is $b_{3 g} \otimes b_{2 u}=b_{1 u}$.

38 H. Köppel, L. S. Cederbaum, W. Domcke and S. S. Shaik, Angew. Chem., Int. Ed., 1983, 22, 210.

39 J. F. Stanton, J. Chem. Phys., 2001, 115, 10382.

40 T. Bally and W. T. Borden, Rev. Comput. Chem., 1999, 13, 1.

41 H. Köppel, W. Domcke and L. S. Cederbaum, Adv. Chem. Phys., 1984, 57, 59.

42 T. Ichino and J. F. Stanton, manuscript in preparation.

43 M. J. Frisch, et al., Gaussian 09, Revision C.01, Gaussian, Inc., Wallingford, CT, 2009 (complete reference given in the ESI $\dagger$ ).

44 J. F. Stanton and J. Gauss, J. Chem. Phys., 1994, 101, 8938.

45 T. Ichino, A. J. Ginaola, W. C. Lineberger and J. F. Stanton, J. Chem. Phys., 2006, 125, 084312.

46 T. Ichino, J. Gauss and J. F. Stanton, J. Chem. Phys., 2009, 130, 174105. 